Géline U r lacher-Becht

B ernadette Litschgi

Sandrine de Raguenel

\title{
Exhumer le Prodigiorum liber de Julius Obsequens à la Renaissance et aujourd'hui : méthodes et enjeux
}

Le Prodigiorum liber (Livre des prodiges) de Julius Obsequens ${ }^{1}$ est une œuvre peu connue, mais qui se prête bien, en raison du caractère limité de sa diffusion, à une réflexion sur la transmission, à des époques diverses, des œuvres antiques voire tardo-antiques, puisque ce Liber date probablement du IVe siècle de notre ère. Il s'agit d'un épitomé, c'est-à-dire d'un abrégé de l'œuvre de Tite-Live comme il en existe d'autres dans la tradition latine ${ }^{2}$, mais qui présente la particularité de ne retenir de l'œuvre du célèbre historien romain que les prodiges relatés par ce dernier. Ces phénomènes inhabituels, que les anciens considéraient comme une manifestation de la volonté divine dont ils pouvaient déduire d'importantes conclusions concernant le passé, le présent et le futur ${ }^{3}$,

* Les recherches sur le Liore des prodiges ont été entreprises par C. Urlacher-Becht. Elles sont présentées ici avec, pour tous les aspects liés au programme Interreg IV, l'éclairage de S. de Raguenel, secrétaire scientifique du projet, et de B. Litschgi, conservatrice co-responsable de l'exposition qui s'est tenue à la Bibliothèque municipale de Mulhouse.

1 L'édition la plus récente est celle d'A. C. Schlesinger parue pour la première fois à Cambridge (Mass.) en 1959 dans la collection de la «Loeb classical library» $\left(\mathrm{n}^{\circ} 404\right)$; il s'agit du quatorzième et dernier volume de l'History of Rome de Tite-Live : Summaries. Fragments. Fulius Obsequens. La traduction française la plus récente, souvent peu littérale, est celle de V. Verger, Les Prodiges de Fulius Obsequens, Paris, Panckoucke, 1842.

2 On pense notamment à l'abrégé de l'Histoire romaine de Florus. Voir, en général, sur les épitomés de Tite-Live, R. Herzog, « Die "Livius-Epitome" and T. Liui periochae omnium librorum $a b$ urbe condita", dans R. Herzog et P.L. Schmidt (dir.), Handbuch der Lateinischen Literatur der Antike, t. V, «Restauration und Erneuerung 284-374 AD », Munich, Beck, 1989, p. 190-193; et en particulier sur le Prodigiorum liber, P. Schmidt, Fulius Obsequens und das Problem der LiviusEpitome. Ein Beitrag zur Geschichte der lateinischen Prodigienliteratur, Wiesbaden, Steiner, 1968.

3 Sur ces phénomènes qui frappèrent grandement l'imagination des anciens en rompant le cours des lois naturelles, voir R. Bloch, Les Prodiges dans l'antiquité classique, Paris, Puf, 1963. 
ont fourni la matière de 77 notices de type annalistique toutes composées selon le même modèle tripartite: nom des consuls en charge l'année en question; récit des prodiges survenus; rappel des événements marquants de la politique intérieure et/ou extérieure ainsi annoncés. Elles s'étendent du consulat de Lucius Scipion et Caius Lélius (190 av. J.-C.) à celui de Paulus Fabius Maximus et de Quintus Elius Tuberon (11 ap. J.-C.), quand l'œuvre de Tite-Live remonte - ainsi que s'en fait l'écho le titre latin, $A b$ Urbe condita - «à la fondation de la Ville » (753 av. J.-C.). Le début, qu'on estime à 10\% environ, a donc disparu 4 .

Cet opuscule fait partie de ces ouvrages qu'on perd périodiquement de vue et qu'on redécouvre à la faveur de hasards divers, d'où son intérêt pour l'histoire de la transmission des textes antiques, en particulier des textes secondaires. En nous focalisant sur deux exemples de telles redécouvertes, celle d'Alde Manuce à l'époque humaniste et celle, bien plus modeste, des universitaires qui se sont efforcés, dans le cadre du projet «Interreg IV Rhin supérieur B 22 », d'exhumer des bibliothèques du Rhin supérieur les éditions humanistes des auteurs grecs et latins, nous aimerions engager une réflexion sur les circonstances de telles remises au jour. Il s'agira d'en mesurer la portée, aussi bien du point de vue de l'œuvre, en étudiant quels aspects en furent précisément remis en valeur, que de celui du public touché, en examinant qui eut connaissance de ces deux redécouvertes et par quelle voie de transmission.

\section{La redécouverte humaniste}

Le Livre des prodiges fut, au sens propre du terme, redécouvert à l'époque humaniste, puisque c'est avec l'édition d'Alde Manuce parue à Venise en 1508 que commence l'histoire de ce texte. De fait, cette édition princeps se fonde sur un unique témoin manuscrit, auquel le célèbre éditeur-imprimeur fut le seul à avoir accès ; tous ses successeurs s'appuient sur son édition ${ }^{5}$. On ignore tout de cette source, sinon qu'il s'agissait d'un présent que lui avait fait Jodocus de Vérone, afin qu'Alde le fît imprimer avec les Épitres de Pline le Jeune, ainsi que le De Viris illustribus in Re militari, et in administranda Republica faussement attribué à Pline, et le De claris Grammaticis et Rhetoribus de Suétone ${ }^{6}$. Cette déclaration permet d'emblée de relativiser l'ampleur de cette redécouverte, car elle montre clairement qu'il s'agit d'une œuvre de commande. Il est du reste significatif

La partie conservée représente, dans les éditions modernes du type Teubner (Leipzig, 1910), une trentaine de pages.

5 Voir le recensement quasi complet d'Alex Nice (Université Libre de Bruxelles) : http://www.alexthenice.com/obsequens/editions/16th.html (consulté le 18 septembre 2016).

6 «Addito etiam Iulii Obsequentis libro de Prodigiis, quem mihi Iucundus meus iucundissimus dono dedit, ut una cum aliis in hoc uolumine imprimendum curarem » [ «J'ai aussi ajouté le livre sur les prodiges de Julius Obsequens que m'a donné le fort agréable Jocondus [l'agréable], afin que je le fasse imprimer avec d'autres dans ce volume »] (Aldus Pius Manutius Romanus Aloisio Mocenico equiti, et senatori Veneto, p. 2, nous traduisons). 
qu'Alde Manuce ne dise, dans sa dédicace, rien de plus au sujet du Prodigiorum liber: ce silence, qui contraste avec le développement de longueur variable consacré aux autres livres édités dans le même ouvrage, en dit paradoxalement long sur l'intérêt limité que devait présenter l'ouvrage à ses yeux.

Les mêmes œuvres firent l'objet de plusieurs rééditions conjointes, qui expliquent la relativement grande diffusion de l'œuvre de Julius Obsequens jusqu'au milieu du XVIe siècle. La nette dépendance de ces éditions à l'égard de l'édition princeps ne fait aucun doute, et est d'ailleurs explicitement revendiquée par la plupart des éditeurs dans leurs écrits liminaires, à l'instar de Beatus Rhenanus (Strasbourg, 1514) ou d'André Cratandre (Bâle, 1521) ${ }^{8}$. Cette dépendance nous incite, d'une autre manière, à relativiser l'intérêt accordé au Livre des prodiges, d'autant plus qu'elle est systématique, alors que les ouvrages édités dans le même volume ont connu une tradition éditoriale indépendante ${ }^{9}$ : à l'évidence, le Liber n'a pas tant retenu l'attention pour lui-même, que par fidélité aux choix éditoriaux de l'édition princeps.

Ce panorama éditorial évolua durant la seconde moitié du XVIe siècle sous l'influence d'un homme, Conrad Lycosthène (1518-1561), qui compte parmi les figures humanistes rhénanes puisqu'il est natif de Rouffach et passa une grande partie de sa vie à Bâle. Il y enseigna la grammaire et la dialectique de 1542 à 1545, avant de devenir diacre de l'église Saint-Bernard. Il continua d'exercer cet office jusqu'à la fin de sa vie, conjointement à ses activités littéraires, dont l'établissement de la première édition indépendante du Prodigiorum liber. Gette dernière fut imprimée à Bâle en 1552, sur les presses du fameux Jean Oporin dont Lycosthène avait épousé la sœur, Chrétienne Herbster, et rééditée à trois reprises jusqu'à la fin du siècle ${ }^{10}$.

7 «Quod enim summopere studiosis curandum est, emendatum esse scito, $\mathcal{E}$ Aldini exemplaris foeturam non adulteram, sed legitimam » [ "Sache en effet que ce dont se sont occupés avec le plus grand soin les érudits a été corrigé, et que la production engendrée à partir de l'exemplaire d'Alde n'a rien d'adultère, mais qu'elle est légitime »] (Strasbourg, M. Schürer, 1514, « Beatus Rhenanus Gaspari Vuesterburgio », p. [1], nous traduisons).

8 «Damus non minus (ut spero) utilitatis adlaturum opusculum, nempe epistolas Plinii Secundi [...], una cum politissimo Panegyrico Traiano Imperatori dicto, et libello de uiris illustribus, ad exemplar Aldinum [...] Adnexis quoque claris Grammaticis, et Rhetoribus Tranquilli, et Iulii Obsequentis Prodigiis » [« Nous publions un modeste ouvrage qui ne sera pas, je l'espère, peu utile, à savoir les épîtres de Pline le Jeune, avec le Panégyrique très raffiné qu'il adressa à l'empereur Trajan, et son petit livre sur les hommes illustres, conformément à l'exemplaire d'Alde [...] S'y ajoutent les illustres grammairiens et rhéteurs de Suétone, ainsi que les prodiges de Julius Obsequens »] (Bâle, A. Cratandre, 1521, Andreas Cratander candido lectori, p. [2], nous traduisons). A. Cratandre explique, dans la suite de son avertissement au lecteur, pourquoi il a jugé utile de reproduire, en outre, la préface d'Alde Manuce, ce qui prouve d'une autre manière sa dette à l'égard de l'édition princeps.

9 Voir par exemple le volume paru à Strasbourg chez M. Schürer, en 1510, qui réunit uniquement C. Plinii Secundi iunioris liber illustrium uirorum et C. Suetonii Tranquilli de grammaticis et rhetoribus claris libellus.

10 Lyon, J. de Tournes et W. Gazeau, 1553 ; Bâle, H. Petri, 1557 ; Lyon, J. de Tournes, 1589. S'ajoutent à ces rééditions deux traductions dans une langue « moderne » : De' Prodigi. Polidoro 
Le déplacement des centres d'intérêt dont témoigne cette édition est net : c'est bien le sujet même du Livre des prodiges qui a retenu l'attention de l'humaniste alsacien, parce que les circonstances dans lesquelles se sont produits ces prodiges et surtout leurs funestes conséquences lui apparaissaient comme un avertissement divin semblable à ceux par lesquels Dieu continuait de mettre en garde les hommes de son époque, sans qu'ils y prêtassent forcément plus attention que les anciens. Cette idée, qui montre quels sont les enjeux spirituels d'une telle édition, est affirmée avec force dans l'épître dédicatoire ; nous nous bornons à en citer le court passage dans lequel Lycosthène explicite clairement son dessein éditorial :

J'ai voulu publier le Prodigiorum liber de Julius Obsequens, dont il n'existait qu'un fragment, pour rapprocher les prodiges des païens de ceux qui se produisent par un effet de la volonté divine de notre temps, et mesurer ensuite, à partir de l'issue des événements, combien ces signes terrifiants annoncent toujours quelque malheur imminent, afin qu'ainsi avertis par l'exemple d'autres [hommes, i.e. les anciens], nous examinions attentivement les moyens d'éviter ces dangers. ${ }^{11}$

Plusieurs autres indices confirment ce grand intérêt de l'humaniste pour les prodiges. Tout d'abord, Conrad Lycosthène a entrepris de restituer le début du Liber, en s'inspirant de la méthode et du style de Julius Obsequens pour inventer de toutes pièces les notices manquantes -54 au total ${ }^{12}$. Ensuite, il a cherché à approfondir la réflexion sur le sujet en lui offrant une sorte de prolongement contemporain, à travers l'ajout, dès la première édition de cette version augmentée en 1552, des trois livres De prodigiis de l'écrivain et historien italien Polydore Virgile (1470-1555), ainsi que des deux livres De ostentis (i.e. De prodigiis) du savant allemand Joachim Camerarius (1500-1574)13. Enfin, il a lui-même entrepris, à peine cinq ans après sa première édition du Prodigiorum liber, la

Vergilio De’ Prodigi libri III, trad. D. Maraffi, Lyon, J. de Tournes, 1554 ; Des prodiges. Plus trois livres de Polydore Vergile sur la mesme matiere, trad. G. de La Bouthière, Lyon, G. de la Bouthière, 1555.

11 "Iulii autem Obsequentis Prodigiorum librum, cuius fragmentum tantum extabat, eo libentius hoc tempore edere uolui, ut gentilium prodigia cum his quae nostro tempore diuinitus eduntur, conferrentur; $\mathcal{E}$ expenderetur deinde ex rerum euentu, horrenda illa signa semper aliquid imminentium malorum hominibus portendisse, quo aliorum tandem exemplo moniti, euitandorum periculorum rationes eo diligentius iniremus » (Praefatio de l'édition de Bâle, J. Oporin, 1552, f. 6ro ${ }^{\circ}$.

12 Voir le titre programmatique de l'édition de 1552: Obsequens, Iulius, Prodigiorum liber, ab Urbe condita usque ad Augustum Casarem, cuius tantum extabat Fragmentum, nunc demum Historiarum beneficio, per Conradum Lycosthenem Rubeaquensem, integritati sua restitutus [...]. Sur la méthode de Lycosthène, voir C. Urlacher-Becht, «Les sources de Conrad Lycosthène dans son édition restituée du Prodigiorum liber de Julius Obsequens », dans M. T. Schettino et C. Urlacher-Becht (dir.), L'Autorité intellectuelle des Anciens : affirmations, appropriations, détournements, Besançon, Presses Universitaires de Franche-Comté, à paraître.

13 Les mêmes ouvrages figurent dans les rééditions ou les traductions de 1553, 1554, 1558, 1589. 
rédaction d'une "chronique » universelle des prodiges, depuis la création du monde jusqu'aux événements singuliers de son époque, afin de démontrer que

Dieu ne cesse de prodiguer à l'homme les témoignages de sa bienveillance comme de sa colère, et qu'en particulier il l'instruit, par des signes, des changements et des malheurs à venir. ${ }^{14}$

Un dernier indice, qui mériterait de faire l'objet d'une étude approfondie, atteste ce déplacement des centres d'intérêt, car il a modifié de manière notable l'approche purement littéraire qui prévalait jusque-là du Livre des prodiges: l'illustration systématique du texte, dès sa première édition augmentée, par un nombre important de gravures sur bois. L'édition bâloise de 1552 en compte 82 au total, dont certaines reviennent à plusieurs reprises, et ce, parfois en l'espace de quelques pages, voire sur deux pages en vis-à-vis. Ce procédé, s'il a pu servir les intérêts de l'imprimeur, est aussi conforme aux intentions de l'éditeur qui entendait montrer que, malgré la variété des lieux, des temps et des circonstances, Dieu ne se lassait pas d'envoyer les mêmes avertissements aux hommes.

Cet intérêt renouvelé, au cours de la seconde moitié du XVI ${ }^{e}$ siècle, pour le Prodigiorum liber de Julius Obsequens est ainsi d'abord celui d'un amateur de prodiges, Conrad Lycosthène : les quatre éditions imprimées entre 1552 et 1589 n'en font en effet qu'une d'un point de vue philologique, puisque le texte latin des éditions de 1553, 1557 et 1589 correspond à celui de l'édition de 1552; quant aux deux traductions dans une langue moderne qui virent le jour peu de temps après la parution de l'édition de Lycosthène (en italien en 1553 et en français en 1555)15, elles se fondent toutes deux sur le texte de cette édition augmentée.

De même que toutes les éditions de la première moitié du XVI ${ }^{e}$ siècle portaient l'empreinte d'Alde Manuce, celles de la seconde moitié portent donc celle de Conrad Lycosthène, à cette différence près que la multiplication des éditions isolées ou des recueils thématiques et le renouvellement éditorial, notamment apporté par l'ajout d'illustrations, laissent transparaître un intérêt pour l'ouvrage en lui-même et manifestent les enjeux religieux sous-jacents. La volonté de rendre cet ouvrage accessible à un plus large public par l'entremise de traductions modernes va dans le même sens, et dans celui d'un «perfectionnement de la langue française », ainsi qu'y insiste le traducteur

14 J. Céard, La Nature et les prodiges, Genève, Droz, 1977, p. 187-188. La chronique en question s'intitule Prodigiorum ac ostentorum chroniconn [...], Bâle, Petrus, 1557. Sur la réception du Prodigiorum liber au XVI siècle, voir M. Laureys, «Die Bewertung der Prodigien und die Rezeption des Julius Obsequens im Humanismus des 16. Jahrhunderts », dans W. Hogrebe (dir.), Mantik. Profile prognostischen Wissens in Wissenschaft und Kultur, Würzbourg, Königshausen \& Neumann, 2005, p. 201-221.

15 Voir supra n. 10. 
français Georges de la Bouthière dans les dernières lignes de son avertissement aux lecteurs ${ }^{16}$. L'effort de transmission dont témoigne cette traduction est d'autant plus notable qu'il passe aussi par l'ajout d'un commentaire relativement fourni qui vise surtout, toujours selon les déclarations liminaires du traducteur, à éclaircir les « dénominations imposées par les Anciens », en particulier celles des «ville, pays, contrée ou territoire», mais aussi les «cérémonies et étranges façons de faire des Anciens ${ }^{17}$ ».

L'enjeu que représentent ainsi les publics-cibles n'est pas propre à la démarche humaniste: il est également au centre de la revalorisation contemporaine des auteurs antiques à laquelle aspira, dans le cadre du programme "Interreg IV Rhin supérieur B 22 », une constellation d'acteurs variés, œuvrant à la médiation de cette redécouverte humaniste.

\section{La redécouverte contemporaine : le programme Interreg IV}

L'objectif du programme Interreg, financé par l'Union Européenne à travers le Fonds Européen de Développement Régional, était de mettre en valeur, auprès du grand public à l'échelle régionale et auprès de la communauté scientifique à l'échelle universitaire, les œuvres antiques imprimées au XVe ou au XVIe siècle, et conservées dans les bibliothèques du Rhin supérieur. Echelonné de mars 2011 à mars 2014, il prit pour emblème une représentation $\mathrm{d}^{\prime}$ Humanitas $^{18}$, une belle jeune femme aux cheveux longs tenant un livre, assise sur un char qui avance sous les efforts conjoints de Cicéron et de Virgile, d'Homère et de Démosthène (Fig. 1). Cette figure allégorique incarnait, suivant les termes de la devise associée, les deux grandes visées de l'humanisme : "une langue plus châtiée et une vie plus honnête » ( Lingua expolitior et uita emendatior »). L'objectif était de s'inscrire, à l'époque contemporaine, dans la continuité du reditus ad fontes (《retour aux sources ») engagé par les humanistes et de mettre en lumière le rôle prépondérant joué à la Renaissance par la région du Rhin supérieur. La riche production aujourd'hui conservée dans les bibliothèques du Rhin supérieur en témoigne ${ }^{19}$.

16 «Il me suffit qu'en ayant fait mon mieux, un chacun entende que j'ay plus pretendu à l'edificacion des simples, amendement de plusieurs abusez, \& avancement de la langue Françoise, que à une ambition affectee de ma propre gloire » (p. [4]).

17 Ibid., p. [3].

18 Cet emblème est extrait de la page de titre des Opera de Sénèque, sortie en juillet 1515 des presses de Johann Froben. L'encadrement de cette page de titre fut utilisé pour la première fois en août 1513: Paulus Cortesius In sententias [...], Bâle, Froben, 1513. La Bibliothèque Humaniste de Sélestat en possède un exemplaire (cote K 866).

19 Voir F. Ritter, Catalogue des Incunables et Livres du XVIe siècle de la Bibliothèque Municipale de Strasbourg, Strasbourg, Heitz \& Cie, 1948 ; C. Reske, Die Buchdrucker des 16. und 17. Fahrhunderts im deutschen Sprachgebiet. Auf der Grundlage des gleichnamigen Werkes von Josef Benzing, Wiesbaden, Harrassowitz, 2007. 
Pour ce faire, plusieurs acteurs se sont constitués «partenaires»: des bibliothèques publiques, comme la Bibliothèque municipale de Mulhouse ou la Bibliothèque Humaniste de Sélestat; des bibliothèques privées, à l'exemple de la Médiathèque protestante de Strasbourg; des bibliothèques universitaires avec d'une part la Bibliothèque nationale universitaire de Strasbourg, et d'autre part deux bibliothèques étrangères ${ }^{20}$, la Bibliothèque universitaire de Fribourg-enBrisgau et la Bibliothèque universitaire de Bâle. Toutes ces bibliothèques ont mis leur fonds ancien à la disposition des chercheurs et ont favorisé ce travail de recherche sur leurs incunables et impressions du XVIe siècle en apportant leurs compétences propres. Du côté universitaire, les universités de Haute-Alsace, de Fribourg et de Strasbourg, déjà unies depuis une dizaine d'années dans le cadre de l'institution Eucor "Collegium Beatus Rhenanus », se sont partagé la responsabilité de ce projet transfrontalier. En collaboration avec l'université de Bâle, elles ont fourni des enseignants-chercheurs, antiquisants ou seiziémistes, qui ont accordé un pourcentage défini de leur temps de travail au projet.

L'engagement conjoint des bibliothèques et des universités a d'abord permis d'identifier le corpus d'étude. De fait, les incunables et premiers imprimés de la région du Rhin supérieur, remarquables par leur nombre et leur qualité, souffraient d'un vrai manque de visibilité : recensement absent ou incomplet, numérisation très partielle... Par le passé, quelques expositions avaient donné l'occasion de les valoriser ${ }^{21}$, mais de nombreux ouvrages restaient inconnus des spécialistes comme du grand public.

Pour les mettre en valeur, différents outils ont été créés. Un site internet présente les travaux réalisés, les expositions en cours et le retentissement médiatique du projet ${ }^{22}$. Une base de données vise à recenser les éditions humanistes des auteurs grecs et latins conservées dans le Rhin supérieur et permet d'en mesurer l'apport ${ }^{23}$. Enfin, sept expositions destinées au «grand public » furent organisées dans les bibliothèques partenaires de mai 2012 à décembre 2013. Les thématiques, toutes différentes, furent définies en fonction de leur fonds ancien propre ${ }^{24}$. Une médiation fut systématiquement proposée

20 L'Allemagne était aussi représentée par la Badische Landesbibliothek de Karlsruhe.

21 Incunables, Bibliothèque municipale de Colmar, 1972; Oberrheinische Buchillustration, Bibliothèque universitaire de Bâle, 1972; L'Humanisme et la réforme à Strasbourg, Ancienne Douane de Strasbourg, 1973; Trésors des Bibliothèques de Colmar et de Sélestat, Eglise des Dominicains de Colmar, 1998; Beautés cachées: sur la route des incunables, Bibliothèque municipale de Strasbourg, 2007.

22 www.humanisme-du-rhin-supérieur.eu

23 Parmi les exemples les plus spectaculaires, on citera les éditions illustrées, et parfois annotées, de Térence (1496), Horace (1498), Boèce (1501) ou encore Virgile (1502), parues chez l'imprimeur strasbourgeois J. Grüninger : on les trouve quasiment dans toutes les bibliothèques du Rhin supérieur, mises en couleur ou non, le cas échéant suivant des techniques et des coloris différents.

24 Le thème des sept expositions organisées entre mai 2012 et décembre 2013 fut le suivant : «Humanisme \& imprimerie » (Haguenau); «Entre Bâle et Sélestat : l'humanisme au XVI siècle » (Bâle); "Culture humaniste des $\mathrm{XV}^{\mathrm{e}}$ et $\mathrm{XVI}$ e siècles à travers les collections 
par le biais de visites guidées, de conférences et d'animations. Par ailleurs, une valise pédagogique fut constituée à l'adresse des enseignants du secondaire : elle leur proposait, en lien avec les expositions, des activités diverses s'appuyant sur des extraits et/ou des reproductions d'ouvrages des $\mathrm{XV}^{\mathrm{e}}$ et XVI ${ }^{\mathrm{e}}$ siècles.

\section{Le Livre des prodiges : enjeux de la redécouverte scientifique}

Les approches diverses auxquelles s'est prêté le Livre des prodiges dans le cadre de ce programme Interreg éclairent bien les enjeux liés à la redécouverte contemporaine des auteurs anciens, en particulier des auteurs mineurs largement déconsidérés face aux « grands classiques ». De fait, d'un point de vue scientifique, Julius Obsequens fait partie de ces auteurs antiques qui ont peu retenu l'attention des chercheurs, d'une part parce qu'il se situe dans l'ombre de sa source littéraire principale, l'historien Tite-Live, d'autre part parce qu'il fait partie de ces auteurs tardifs auxquels on a, jusqu'à une époque récente, dénié tout intérêt ${ }^{25}$. Ce mépris est très net si l'on fait le bilan de la bibliographie moderne ${ }^{26}$.

Il n'existait ainsi aucune étude exhaustive de la tradition éditoriale de Julius Obsequens: ses éditeurs comme les études générales sur la transmission des textes antiques y consacrent à peine quelques lignes, sans prendre en considération l'ensemble des éditions dont on peine à trouver une liste complète ${ }^{27}$. Même si toutes les éditions des XVe et XVIe siècles ne sont pas représentées dans les bibliothèques du Rhin supérieur, c'est néanmoins le cas de la plupart d'entre elles ; la notice complétée pour chaque ouvrage, en particulier la troisième partie dédiée à la transmission et la réception de chaque édition ${ }^{28}$, constitue de ce fait un net progrès.

mulhousiennes » (Mulhouse); "L’humanisme rhénan aux $\mathrm{XV}^{\mathrm{e}}$ et $\mathrm{XVI}$ e siècles entre héritage antique et religion: Ludwig Ber, théologien ami d'Érasme, et autres lettrés " (Colmar); "Humanisme rhénan et géographie : Martin Waldseemüller et la découverte du Nouveau Monde » (Fribourg-en-Brisgau); « Matthias Schürer de Sélestat, imprimeur du XVIe siècle et citoyen de la République des lettres» (Sélestat); «Ottmar Nachtgall : quand Strasbourg chantait l'humanisme » (Strasbourg).

25 C'est la thèse désormais révolue de la « décadence » romaine, y compris sur un plan littéraire : voir le titre polémique d'H.-I. Marrou, Décadence romaine ou Antiquité tardive? III ${ }^{e-V I}$ siècle, Paris, Éd. du Seuil, 1977.

26 Les études sont rares et, pour la plupart, fort indigentes : voir la bibliographie générale (sur Julius Obsequens, mais aussi les prodiges et les épitomés de Tite-Live) mise en ligne par Alex Nice : http://www.alexthenice.com/obsequens/bibliography.html.

27 Voir par exemple les huit lignes à peine consacrées à «Julius Obsequens » dans L. Reynolds et al. (dir.), Texts and Transmission. A Survey of the Latin Classics, Oxford, Clarendon Press, 1986, p. 52.

28 La notice en question est composée de cinq parties : la première donne les renseignements bibliographiques habituels (auteur, titre complet, colophon, lieu et date d'impression, imprimeur etc.); la deuxième décrit les contributions humanistes de l'ouvrage (épître dédicatoire, avis aux lecteurs, commentaires etc.) ; la troisième est dédiée à la transmission et à 
L'enquête menée dans chacune des bibliothèques partenaires du projet a facilité l'accès aux fonds anciens qui seront, à terme, répertoriés pour l'ensemble des bibliothèques dans une base de données unique. Pour prendre l'exemple de la Bibliothèque municipale de Colmar, toute recherche bibliographique passe, nécessairement, par la consultation d'anciens catalogues manuscrits rouges, uniquement accessibles sur place, qui requièrent un laborieux déchiffrage. De même, si la Bibliothèque nationale universitaire de Strasbourg dispose de catalogues plus complets et aisément accessibles par internet, il faut encore se référer auxdits "registres noirs ${ }^{29}$ ", en cours de rétro-conversion, pour les acquisitions antérieures à 1920. Or ces catalogues ne sont pas sans comporter des erreurs et ne permettent guère de repérer certaines anomalies notamment liées à la transmission conjointe, durant la première moitié du XVIe siècle, de l'œuvre de Pline et de Julius Obsequens.

En outre, le programme Interreg a mis en lumière l'importance des annotations manuscrites datant de l'époque humaniste qui font tout l'intérêt des ouvrages parfois conservés à l'identique dans plusieurs bibliothèques de la région. Elles font, dans la notice complétée par les enseignants-chercheurs, l'objet d'un examen spécifique dans la partie dédiée à l'exemplarisation de chaque volume conservé dans les bibliothèques partenaires. Or ces annotations fournissent, à maintes reprises, des renseignements précieux sur la «société du livre » au XVIe siècle, en particulier la nature des ouvrages qu'échangeaient les humanistes locaux ainsi que la constitution de leur bibliothèque personnelle. Les visiteurs de l'exposition de Colmar en ont eu un exemple très concret à travers l'immersion dans la bibliothèque personnelle de Ludwig Ber, qui fit partie des théologiens ouverts à la culture humaniste. On notera d'ailleurs que cet humaniste possédait, lui aussi, un exemplaire du Prodigiorum liber qui n'est pas répertorié sous le nom de Julius Obsequens dans les catalogues, car il s'agit d'un volume comprenant aussi et surtout les Épitres de Pline: il fut, de ce fait, répertorié sous le nom de l'épistolier latin.

Parmi les illustres possesseurs représentés dans le fonds colmarien figurent également Augustin Gynzter, qu'on peut probablement identifier au secrétaire de l'ambassadeur de Habsbourg ${ }^{30}$, et Johann Leglin, un proche de Calvin, qui possédait un exemplaire de l'édition de 1514 qu'il a copieusement annoté, sans que ces annotations soient cependant toujours liées aux œuvres imprimées, puisqu'il y a notamment copié le texte de plusieurs fables: dans le cas du Prodigiorum liber, il s'est contenté de reproduire, en marge, les termes clés de l'opuscule et d'expliciter certaines expressions au moyen de synonymes ${ }^{31}$. Ces

la réception de l'édition; la quatrième à l'exemplarisation de chaque impression conservée dans l'une des bibliothèques partenaires (localisation, cote, ex-libris, annotations); la cinquième propose, enfin, une bibliographie récente.

29 Voir http://www.bnu.fr/catalogues/les-registres-noirs (consulté le 18 septembre 2016).

30 Colmar, Bibliothèque municipale, P 1017 (Paris, Gourmont, 1511).

31 Colmar, Bibliothèque municipale, P 10520 (Strasbourg, Schürer, 1514). 
annotations donnent des indications précieuses sur l'intérêt que trouvaient les humanistes à la lecture des œuvres antiques. Leur absence même est significative, car elle témoigne du relatif désintérêt pour les ouvrages en question : c'est le cas dans la plupart des éditions de Julius Obsequens qui datent de la première moitié du XVIe siècle, moins richement annotées que celles d'auteurs plus fameux.

\section{Faire découvrir Julius Obsequens au " grand public »}

Les enjeux scientifiques du programme, qui ont abouti à la création d'un outil informatique original, facilitant l'accès aux éditions d'auteurs antiques imprimées à la Renaissance, diffèrent radicalement des enjeux liés au «grand public ». Il ne s'agit plus alors d'un public spécialisé, pour ainsi dire conquis d'avance, mais d'un public très hétérogène, parfois largement étranger au patrimoine antique et humaniste. Pour l'atteindre, d'autres moyens de médiation furent déployés, notamment dans le cadre des expositions organisées dans les bibliothèques partenaires.

D'un point de vue général, ces expositions visaient à mettre en valeur des ouvrages et des hommes emblématiques des $\mathrm{XV}^{\text {e }}$ et $\mathrm{XVI}$ e siècles, à la croisée d'un événement, l'invention de l'imprimerie, et d'un mouvement culturel, l'humanisme, en particulier rhénan. Cet aspect transfrontalier du sujet a d'ailleurs conduit à la conception des panneaux, des cartels et des catalogues en version bilingue pour permettre une réception plus étendue et nourrir les échanges. Ces projets articulaient, comme on l'a vu, deux approches et deux pratiques professionnelles - celle des universitaires, tournée vers l'érudition et l'exhaustivité, et celle des bibliothécaires, soucieux de toucher un public plus large ; aussi s'agissait-il de délivrer un contenu scientifique sans tomber dans l'élitisme. Leurs motivations communes étaient de faire découvrir au grand public les collections humanistes, de mettre en lumière les spécificités de l'humanisme rhénan, d'explorer l'histoire du livre à travers des moments charnières (passage du livre médiéval à l'imprimé, apparition de la gravure d'illustration, diversification de l'édition) ; enfin, de valoriser le patrimoine humaniste de la région. L'un des enjeux majeurs de la conception des différentes expositions résidait dans une habile concertation entre érudition et scénographie, qui apporte un éclairage digne d'intérêt sur les conditions d'une plus large transmission du patrimoine antique et humaniste à l'époque contemporaine.

Au plan formel, chaque exposition reposait sur un double dispositif constitué de panneaux d'information et de ressources documentaires issues des différentes bibliothèques. A Mulhouse, la première démarche a consisté à identifier les ouvrages les plus emblématiques du corpus humaniste et à repérer les éditions remarquables. Cette opération a également permis de prendre connaissance des sujets spécifiques qui, du fait de l'histoire des collections, reflétaient une 
dominante scientifique et artistique. Des développements spécifiques ont ainsi été consacrés à la diffusion des sciences, aux arts figuratifs et à l'imprimerie mulhousienne en complément à la trame commune à l'ensemble du projet. L'étape suivante a consisté à réaliser un parcours et à le mettre en scène en tenant compte des contraintes spatiales. Ainsi, le début du cheminement à proximité de la banque d'accueil a été matérialisé par une grande affiche et un téléviseur diffusant le documentaire réalisé par Interreg. Il se poursuivait ensuite à travers les $120 \mathrm{~m}^{2}$ de la salle d'exposition, s'appuyant sur le discours des panneaux et l'illustration d'une trentaine de pièces présentées sous vitrines ou sous cadres (Fig. 2).

La scénographie a cherché à servir le propos par une approche claire, cohérente et élégante, déclinant des valeurs elles-mêmes intrinsèques à l'humanisme. Il s'est d'abord agi de rendre lisible le propos à travers des sections thématiques et chronologiques claires définies par les panneaux et les vitrines. Ensuite, l'attention a porté sur la mise en valeur des imprimés dans le souci de bien mettre l'accent sur leur double nature d'œuvre et d'ouvrage. Ainsi, des lutrins confectionnés par les relieurs ont servi d'écrin aux livres, et de petits supports glissés sous les livres ont contribué à les mettre en mouvement. Sorties du Cabinet des estampes, des gravures de Schongauer, Dürer et Aldegrever ont également dynamisé la présentation et fait apparaitre le rôle essentiel de l'illustration qui émerge dès le XVe siècle (Fig. 3). Quant à l'éclairage, il a fait l'objet d'un soin particulier pour «mettre en lumière » ce patrimoine exceptionnel. Des visites pour les scolaires et le grand public ainsi que des conférences ont constitué le dernier volet du dispositif de médiation.

Le traitement du Livre des prodiges offre un exemple concret de la manière dont furent opérés ces choix. Au début du travail, lors de la conception de la première exposition qui s'est tenue à la médiathèque de Haguenau, les visiteurs adultes ne firent pas partie du «public cible ». Il s'agissait alors, en marge du thème central de l'exposition («Humanisme et imprimerie aux XVe et XVI ${ }^{e}$ siècles »), de consacrer quelques affiches spécifiques à des ouvrages qui pouvaient intéresser les jeunes visiteurs par leur thème, leur histoire et l'entreprise éditoriale dont elles firent l'objet à la Renaissance, et de leur proposer des activités créatives liées pour les sensibiliser, par le biais des arts plastiques entre autres, à ces œuvres ${ }^{32}$. Les lecteurs plus âgés ont néanmoins été intégrés au projet au fur et à mesure de son avancée, et sont même devenus les principaux destinataires des affiches. Celles-ci furent volontairement diffusées dans plusieurs espaces - entrée, salle de prêt, service jeunesse - en complément du dispositif d'exposition pour toucher les différents publics. Ces outils étaient en effet destinés à délivrer un discours particulièrement adapté aux visiteurs moins initiés et les inviter, par ce biais, à découvrir l'exposition.

32 Les affiches évoquées furent présentées lors des expositions de Haguenau, de Mulhouse et de Colmar. 
Les activités créatives proposées aux enfants dans le cadre des expositions qui se sont tenues à Haguenau et à Mulhouse se fondent sur la séduction exercée non seulement par les illustrations, mais aussi par le thème traité par Julius Obsequens: les prodiges, a fortiori les monstres, n'ont pas fini de fasciner les consciences enfantines (Fig. 4 et 5). De là, deux activités graphiques (avec, à Haguenau, la possibilité d'exposer son «œuvre» sur des panneaux vierges réservés à cet effet): d'une part une activité de coloriage, le but étant, comme cela s'est fait dans les premières éditions imprimées, en particulier celles de l'imprimeur strasbourgeois Jean Grüninger, de mettre en couleur les gravures de l'édition de 1552 ; d'autre part une activité de création proposant aux jeunes enfants, sur le modèle des "monstres » évoqués par Julius Obsequens, d'imaginer à leur tour d'autres créatures fantastiques.

Les pistes pédagogiques liées au Prodigiorum liber firent l'objet d'une présentation approfondie dans la mallette pédagogique mise à disposition de tous les enseignants intéressés par le projet. Elle comprenait, outre le texte de l'ensemble des panneaux réalisés, divers documents exploitables en cours (avec des supports pour les élèves directement reproductibles ainsi que des éléments de réponse fournis séparément). Presque tous ces documents, à l'exception des illustrations mises à disposition en vue de diverses activités créatives, étaient destinés à des enseignants de latin exerçant en lycée, puisque la principale piste suggérée consistait en un examen approfondi de l'exercice de réécriture auquel s'est livré Conrad Lycosthène pour combler les lacunes du texte édité par Alde Manuce ${ }^{33}$. Il s'agissait, dans un premier temps, d'étudier la manière dont Julius Obsequens a trouvé et mis en forme la matière des notices authentiques, pour mieux évaluer ensuite la pertinence de la réécriture de Conrad Lycosthène, que ce soit du point de vue du style, fidèle à celui des chroniques, ou des sources employées. A bien des reprises, cet amateur de prodiges s'est en effet écarté du modèle livien, n'hésitant pas à emprunter sa matière à d'autres sources antiques plus riches, voire à l'œuvre de l'un de ses contemporains... qu'il cite sans le nommer. L'idée était donc de miser sur l'étude de Tite-Live, inscrite au programme des classes de seconde et de première, pour inciter les enseignants à engager une réflexion sur la méthode de l'un de ses abréviateurs tardo-antiques, et leur faire découvrir, à partir de là, le "fabuleux destin » que connut son œuvre ${ }^{34}$ : une longue période d'oubli avant d'être partiellement redécouverte à l'époque humaniste et rééditée sous une forme prétendument achevée par un humaniste qui aimait passionnément les prodiges, au point de dénaturer le projet historique

33 Voir C. Urlacher-Becht, «Les prodiges de l'Histoire romaine de Tite-Live : de l'épitomiste latin Julius Obsequens à son éditeur humaniste Conrad Lycosthène. Quelques pistes pédagogiques (latin, 2nde)», dans «Dossier d'étude pour l'enseignement des langues anciennes », Bulletin CNARELA-ARELAS, n ${ }^{\circ} 35,2015$, p. 26-40.

34 Le titre de la série de posters était: «Le fabuleux destin du Livre des prodiges de Julius Obsequens ». 
de Julius Obsequens en puisant la matière de ses notices à des sources variées, y compris humanistes.

Que peut-on conclure de l'examen de ces deux redécouvertes sur les enjeux de la transmission du Prodigiorum liber de Julius Obsequens à l'époque humaniste et à l'époque contemporaine? Le Livre des prodiges est et restera, une œuvre secondaire, ce qui exclut, de fait, à toute époque une diffusion à grande échelle : la préférence des érudits humanistes comme des universitaires d'aujourd'hui va à l'œuvre originale de Tite-Live, à la source de la compilation. Les rares exceptions concernent des intellectuels férus de prodiges, comme ce fut le cas de Conrad Lycosthène. À l'époque contemporaine, il pourrait, dans la même optique, être intéressant d'engager une réflexion - inédite à notre connaissance - sur ce que nous apprend une telle compilation sur l'arrière-plan culturel et religieux du IVe siècle (un siècle d'anxiété, selon le titre fameux d'Eric Robertson Dodds ${ }^{35}$, mais aussi un siècle où le christianisme l'emporta définitivement sur le paganisme, ce qui pose la question des enjeux spirituels d'un tel opuscule).

Si les antiquisants constituent le premier public d'une telle œuvre, car eux seuls auront les compétences nécessaires pour apprécier le rapport à l'original ou engager une réflexion sur ses implications idéologiques, elle n'est pas vouée, cependant, à demeurer totalement inaccessible à un public non averti, car le sujet abordé et le traitement en images dont il a fait l'objet au cours de la seconde moitié du XVI ${ }^{e}$ siècle continuent de séduire. Comme le rappelle Solas Boncompagni, dans la préface de l'unique traduction contemporaine qui comprend, significativement, la reproduction de plusieurs gravures agrémentant l'édition de 1552, nous ne cessons d'être frappés par les « choses merveilleuses » et les « étranges phénomènes naturels 36 ».

\author{
Géline Urlacher-Becht \\ Université de Haute-Alsace \\ Bernadette Litschgi \\ Bibliothèques-Médiathèques de Mulhouse \\ Sandrine de Raguenel \\ Université de Haute-Alsace
}

35 E. R. Dodds, Païens et chrétiens dans un âge d'angoisse : aspects de l'expérience religieuse de Marc-Aurèle à Constantin, trad. H.D. Saffrey, Paris, La Pensée sauvage, 2010.

36 Il libro dei prodigi, trad. S. Boncampagni, Florence, Edizioni Mediterranee, 1976. 


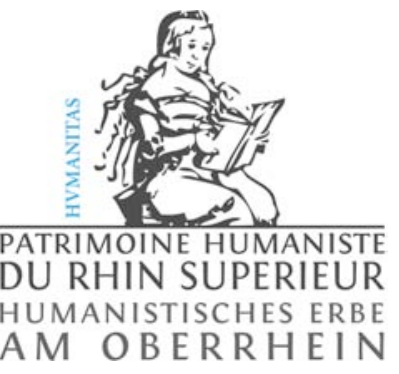

Fig. 1. Auto-portant (C) PHRS

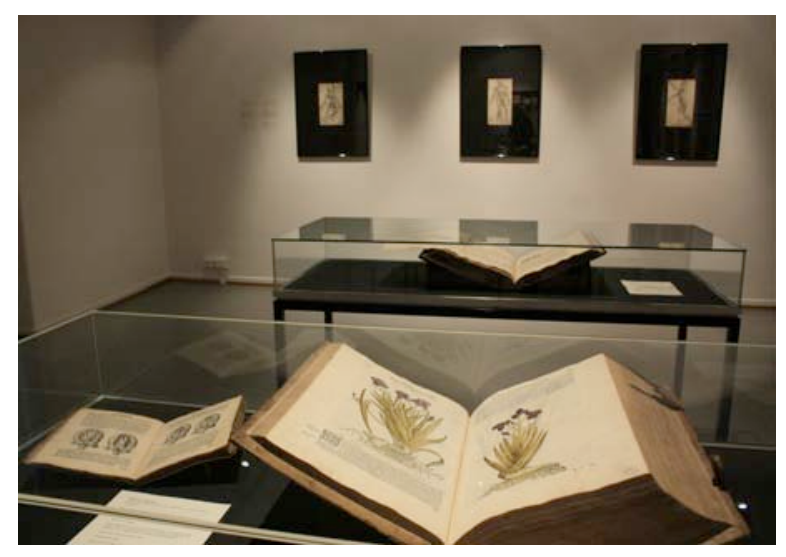

Fig. 2. Exposition « Culture humaniste des XVe et XVIe siècles à travers les collections mulhousiennes » (Bibl. municipale de Mulhouse, juillet- septembre 2012) C BM Mulhouse

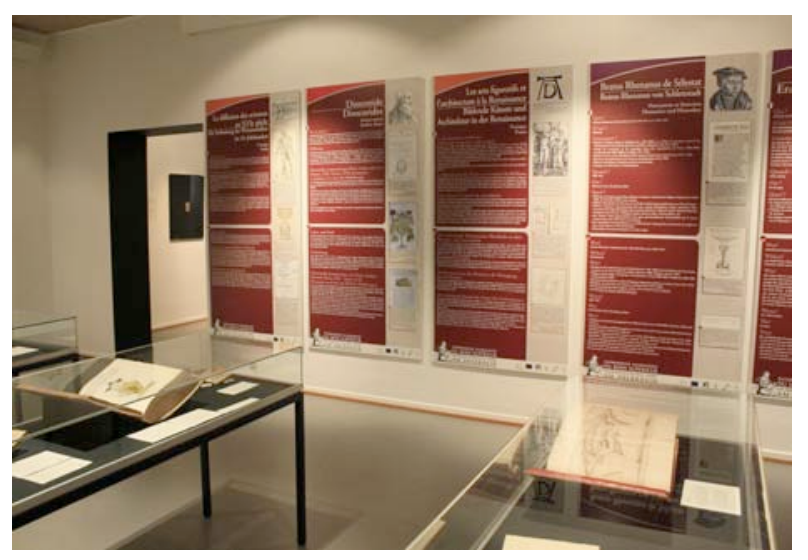

Fig. 3. Exposition « Culture humaniste des XVe et XVIe siècles à travers les collections mulhousiennes » (Bibl. municipale de Mulhouse, juillet- septembre 2012) (C) BM Mulhouse 


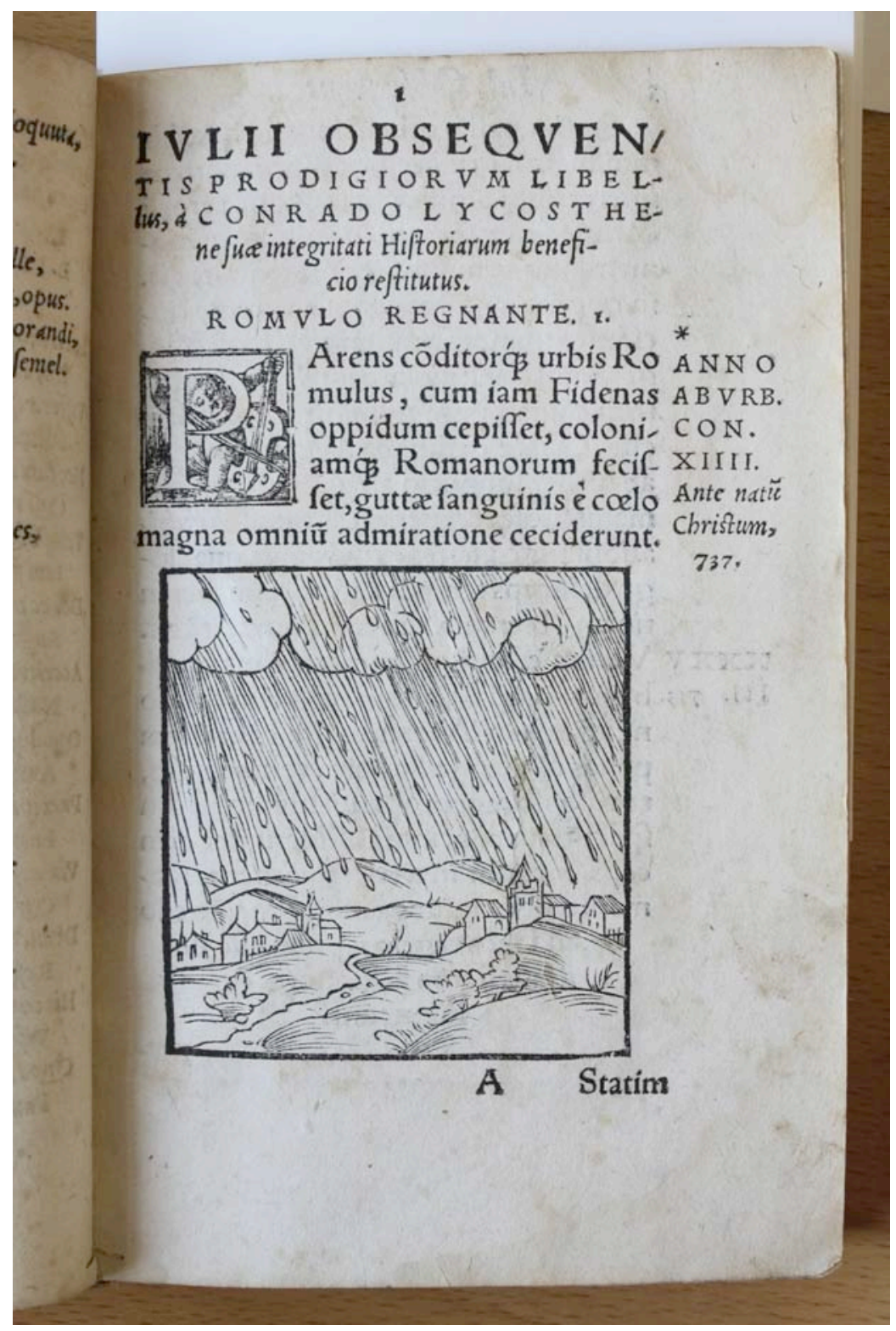

Fig. 4. Prodigiorum liber, éd. Conrad Lycosthène, Bâle, J. Oporin, 1552, p. 1 (C) BM Mulhouse 


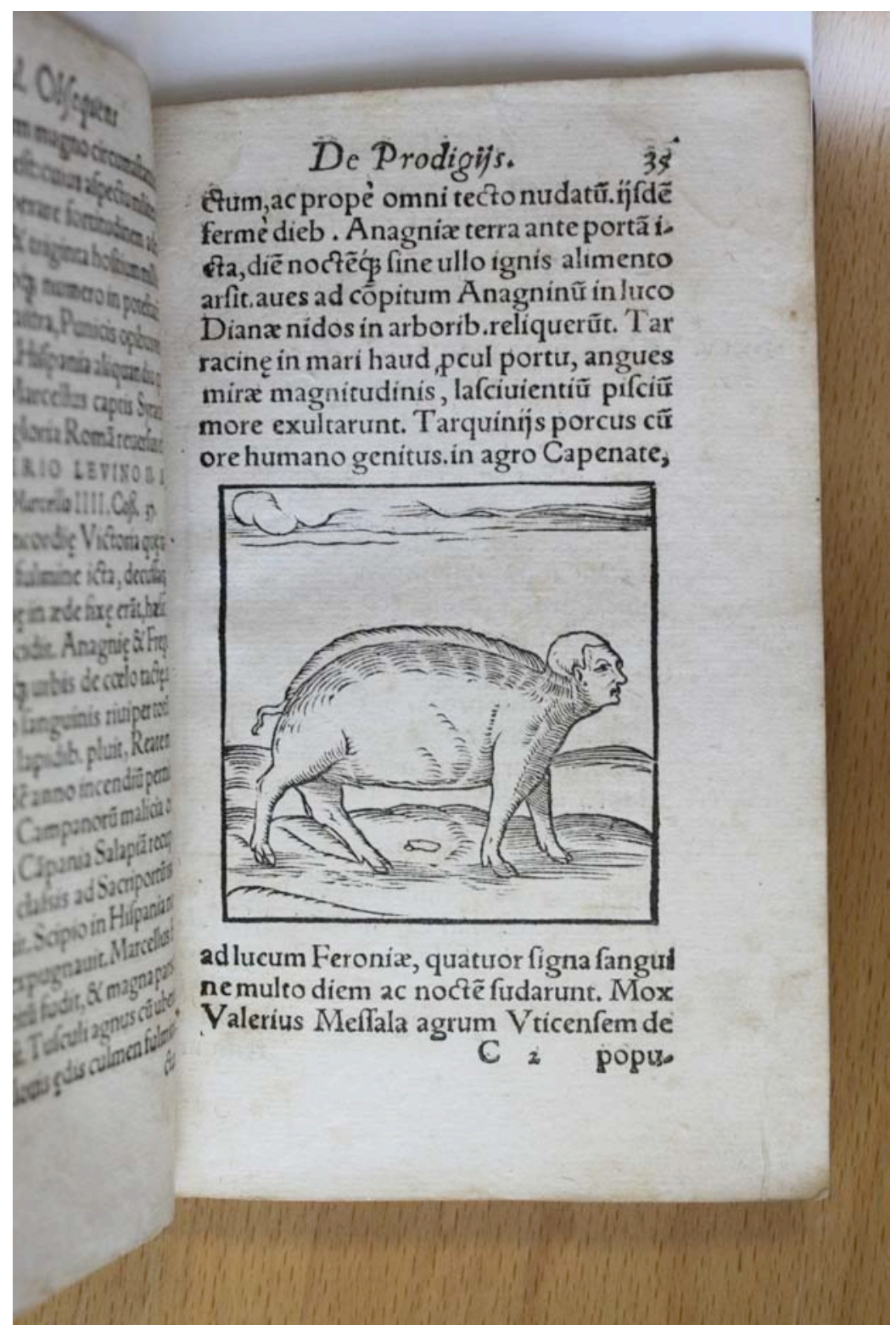

Fig. 5. Prodigiorum liber, éd. Conrad Lycosthène, Bâle, J. Oporin, 1552, p. 35 (C) BM Mulhouse 